\title{
Bildungslandschaften gestalten
}

\section{Für ein kommunal verantwortetes Gesamtkonzept von Bildung, Erziehung und Betreuung}

\author{
Josef Faltermeier und Petra Mund
}

Der Schlüssel für eine erfolgreiche gesellschaftliche Integration junger Menschen liegt in der Bildung. Je früher Kinder gleiche Bildungschancen erhalten, umso weniger können sich durch Lebenslagen bedingte nachteilige Strukturen verfestigen und die soziale Herkunft die Bildungsbiografie von Kindern bestimmen. Deshalb sind die für die Sicherung guter und erfolgreicher Bildungsbedingungen für alle junge Menschen verantwortlichen Institutionen mehr denn je auf Kooperation und Vernetzung angewiesen.

Familien, so die übereinstimmenden Thesen der Kinder- und Jugendberichte der Bundesregierung (1), können die aus den gestiegenen Anforderungen an eine gute Bildung resultierenden Erziehungs- und Bildungsansprüche nicht mehr alleine bewältigen. Sozialer Kompetenzerwerb und schulischer Bildungserfolg ist jungen Menschen heute vielfach nur möglich, wenn sozialstaatliche Institutionen die Erziehungsleistungen in den Familien flankieren - und zwar nicht nur als familiärer Defizitausgleich, sondern als sozialstaatliche Serviceleistungen.

\section{Kommunale Bildungsland- schaften - So kann es gehen}

Vor diesem Hintergrund sind Familie, Schule, Jugendhilfe, Betriebe - mithin alle vor Ort relevanten Bildungsakteure - mehr denn je gefordert, auf eine neue und intelligente Weise zusammenzuarbeiten. In "Kommunalen Bildungslandschaften « sollen diese für das Aufwachsen und die künftigen Chancen junger Menschen entscheidenden Institutionen verbindlich und effektiv zusammengeführt werden. In diesem Beitrag werden, ausgehend von einem erweiterten Bildungsbegriff, die zentralen Merkmale Kommunaler Bildungslandschaften beschrieben, die Anforderungen an die beteiligten Akteure benannt und Wege der institutionellen Vernetzung aufgezeigt. (2)

Ausgelöst durch Debatten über die Ergebnisse der PISA-Studien hat sich in den letzten Jahren deutlich herauskristallisiert, dass Bildung mehr ist als Schule. Bildung in der Wissensgesellschaft muss verstanden werden, als umfassender Lernund Entwicklungsprozess zur Handlungsfähigkeit und Gesellschaftsfähigkeit des jungen Menschen. Schulisches, soziales und emotionales Lernen - oder, wie die EU-Kommission in ihrem Bericht (2002) dies ausdrückt, formales, non-formales und informelles Lernen - stehen in einem engen Zusammenhang, denn ohne ausreichende soziale Schlüsselkompetenzen ist auch die schulische Lernentwicklung nur begrenzt möglich.

An diesem erweiterten Bildungsverständnis setzen Kommunale Bildungslandschaften an. Sie sind ein Referenzrahmen für ein kommunal verantwortetes Gesamtkonzept von Bildung, Erziehung und Betreuung. Sie wollen für alle Bürgerinnen und Bürger die auf örtlicher Ebene vorhandenen Bildungsressourcen systematisch zusammenführen, neue Bildungsressourcen gemeinsam erschließen und sie größtmöglich und effektiv nutzbar machen.

In Kommunalen Bildungslandschaften werden auf der Grundlage gemeinsamer Vereinbarungen mit allen örtlichen bildungsrelevanten Institutionen - Familie, Betriebe, Jugendhilfe, Schule, Volkshochschule, Kindertageseinrichtungen etc. die Verfügbarkeit und Verschränkung dieser Netzwerke vor Ort sichergestellt und damit zu einem kommunalen Gesamtkonzept von Erziehung, Bildung und Betreuung zusammengefügt. Durch die Verzahnung der unterschiedlichen Bildungsakteure werden gleichermaßen auch die verschiedenen Lernwelten und Lernebenen miteinander verbunden.

In der heutigen Praxis stehen die verschiedenen Bildungs- und Lernebenen wie Familie, Kindertageseinrichtungen, Schule, außerschulische Freizeitangebote und Betriebe nach wie vor weitgehend unabhängig und versäult nebeneinander. Dieses Nebeneinander soll überwunden 
und dadurch Synergieeffekte freigesetzt werden. Kommunale Bildungslandschaften wollen - alters- und entwicklungsentsprechend - durch eine Zusammenführung der verschiedenen Bildungskontexte von Kindern und Jugendlichen insbesondere an lebensphasen- oder zielgruppenbezogenen Schnittstellen die Übergänge harmonisieren, so dass Bildungserfolge sich unabhängig von der sozialen Herkunft einstellen können.

Konkret könnte eine Kommunale Bildungslandschaft so aussehen: Kinder kommen im Verlauf ihrer Entwicklung zunehmend mit neuen und differenzierten zukommen muss, nämlich: Eltern und $\mathrm{Fa}-$ milie, dem Elementarbereich, die Schule sowie Ausbildung und Beruf.

\section{Eltern und Familie}

Eltern sind die zentralen Akteure in Kommunalen Bildungslandschaften, sie sind diejenigen, die am besten über die Kompetenzen und Bedürfnisse ihrer Kinder Auskunft geben können. Die Bedeutung von Eltern als Erziehungspartner ist in pädagogischen Institutionen lange unterschätzt worden, ist aber vor dem

\section{»Kommunale Bildungslandschaften sind die Neujustierung der Angebote von Bildung, Betreuung und Erziehung"}

Einrichtungen und Lebenswelten zusammen, die sie bilden und beeinflussen. Schon ab dem ersten Lebensjahr könnte eine kooperative Plattform von Familie, Krippe, Tagespflege, Kindertageseinrichtung, Jugendarbeit, Familienbildung, Migrationsdienst und Gesundheitsdienst die Entwicklung und Persönlichkeitsbildung der Kinder enorm bereichern, wenn zwischen den Institutionen ein abgestimmtes, für jede Familie gut erreichbares und verbindliches Netzwerk der Institutionen und Angebote vorhanden wäre.

Diese kooperative Plattform, also eine "Kommunale Bildungslandschaft «, erweitert sich mit zunehmendem Alter der Kinder: Es kommen Schule, Volkshochschule und andere Bildungsträger, Familienbildungs- und Elternbildungseinrichtungen, Migrationsdienste, Jugendzentren, soziale Bildungskurse, außerschulische Jugendbildung, berufliche Bildung, Wirtschaft und Unternehmen in Betracht.

Schließlich erreicht die kommunale Bildungslandschaft dann eine besondere Qualität, wenn alle lebensalter- und lebensphasenbezogenen Angebote konkret miteinander verschränkt sind. Am Beispiel der Vernetzung der Bildungsangebote für Kinder und Jugendliche wird deutlich, welchen Akteuren und Handlungsfeldern eine besondere Aufmerksamkeit
Hintergrund der Anforderungen eines erweiterten Bildungsauftrags zu einem unumgänglichen professionellen Standard geworden.

Grundbedingung im Umgang und in der Arbeit mit den Eltern ist eine ihnen gegenüber wertschätzende, anerkennende und ressourcenorientierte Haltung von Fachkräften. Nur in einem gleichberechtigten Dialog können der Kontakt und das Vertrauen zwischen Fachkräften und Eltern verbessert werden.

Auf diesem Wege werden Eltern darin unterstützt, von Beginn an die $\mathrm{Zu}$ sammenarbeit mit den unterschiedlichen Institutionen, Kindertageseinrichtungen und Schulen, aktiv zu gestalten. Durch die systematische Einbeziehung von Angeboten der Familienbildung wird die Planung und Organisation von spezifischen Angeboten und Leistungen mit Blick auf die Lebenslagen von Familien und ihren Bedarfen möglich.

\section{Elementarbereich}

Angebote der Kindertagesbetreuung sind, neben von Eltern und Kind gemeinsam wahrgenommenen Angeboten, die ersten Bildungs-, Erziehungs- und Betreuungsangebote außerhalb der Familie. Bedingt durch die zunehmende Heteroge- nität der familialen Lebenswelten und Lebenslagen treten die Kinder mit sehr unterschiedlichen Erfahrungen in den Elementarbereich ein. Dies ist von den Fachkräften zu berücksichtigen.

Der Übergang in diese Einrichtungen stellt dabei für alle Beteiligten einen Schritt von zentraler Bedeutung dar. Ein positives Erleben dieses ersten Übergangs kann als eine Weichenstellung für das Erleben weiterer Übergänge betrachtet werden.

Kindertageseinrichtungen übernehmen mit Blick auf die Weiterentwicklung zu Kommunikations- und Begegnungszentren eine wichtige Rolle zur Unterstützung und Förderung von Familien und leisten ihren Beitrag zur sozialen Integration und Chancengleichheit. Insgesamt muss es Ziel der Bemühungen sein, eine tragfeste Erziehungspartnerschaft zwischen den Familien und den Fachkräften der Kindertageseinrichtungen und Kindertagespflege aufzubauen.

Die frühe Bildungsförderung im Bereich der Kindertagesbetreuung soll altersgemäße Ansätze wählen und unter Beachtung individueller Entwicklungsbedingungen die Anschlussfähigkeit zum Primarbereich der Schule im Blick haben. Dabei ist das Zusammenwirken mit den Eltern eine wichtige Voraussetzung, damit die erfolgreiche Gestaltung von Bildungsbiografien gelingt und gemeinsam mit den pädagogischen Kräften des Elementarbereiches der Schule die Anschlussfähigkeit erreicht wird.

Hilfreich um gerade in dieser besonderen Schuleingangsphase eine Institutionen übergreifende Erziehungspartnerschaft herzustellen sind beispielsweise verbindliche Kooperationsformen, wie regelmäßige Schnupperbesuche in den Grundschulen, gemeinsame Feste und Elternabende, aber auch gemeinsame Fortbildungsveranstaltungen der Fachkräfte.

\section{Schule}

Die Schule ist der erste formale Ort in der Bildungsbiografie junger Menschen. Ihren Bildungs- und Erziehungs- und Betreuungsauftrag kann die Schule jedoch nur in einem weiteren Ausbau ganztägiger Bildungs-, Erziehungs- und Betreuungsangebote realisieren. Die Einbeziehung der Ressourcen und Kompetenzen der Kinder- und Jugendhilfe ist hier insbesondere 
unter qualitativen Gesichtspunkten zwingend geboten. Ganztägige Angebote an der Schule unter Einbeziehung der Kinderund Jugendhilfe dürfen jedoch nicht erneut zu einer Zuweisung separierender Zuständigkeiten führen, am Vormittag die Bildung durch die Schule, am Nachmittag die Betreuung durch die Kinderund Jugendhilfe. Vielmehr gilt es, auf der Basis eines gemeinsamen bildungspolitischen Gesamtkonzeptes, eine Veränderung in der Angebotsstruktur und der Kooperation zu entwickeln.

\section{Ausbildung und Beruf}

Der Wechsel von der Schule in das Erwerbsleben ist in der Bildungsbiografie junger Menschen ein weiterer Übergang von zentraler Bedeutung. Nach wie vor sind die Angebote der beruflichen Bildung und die der Schule inhaltlich zwar eng miteinander verbunden, jedoch nicht immer optimal miteinander verzahnt.

Durch die frühzeitige Vernetzung der Angebote der beruflichen Bildung, der Jugendsozialarbeit, der Schule und der Wirtschaft innerhalb der Kommunalen Bildungslandschaft wird gleichfalls dieser Übergang harmonisiert. Die jungen Menschen werden in ihrer Ausbildungsreife und Berufsorientierung gestärkt. Das Wissen um die wechselseitigen Bedürfnisse der jeweiligen Partner ermöglicht die Entwicklung bedarfsgerechter Angebote für die individuelle Förderung der jungen Menschen.

\section{Schlussbemerkungen}

Um Kommunale Bildungslandschaften aufzubauen und zu gestalten, ist der Rückgriff auf (zumeist bereits vorhandene) gesetzliche Grundlagen, Rahmenbedingungen (bestehende Netzwerke oder Arbeitsgemeinschaften), Planungs- und Steuerungsinstrumente hilfreich und sinnvoll, da ihre Berücksichtigung in der Regel geübte Praxis ist und »lediglich « ihre systematische Verzahnung erfolgreich umgesetzt werden muss. Ausgehend von sozialräumlich ausgerichteten Bedarfen gilt es, konkrete Ziele und Verantwortlichkeiten der einzelnen Akteure und Handlungsfelder zu definieren.

Die Verantwortung für die Bedarfsanalyse, die Entwicklung und die Umsetzung des bildungspolitischen Gesamtkonzeptes liegt in der Hand der Kommune. Von ihr geht die Initiative für den Beginn des Aufbaus Kommunaler Bildungslandschaften aus. Sie stellt ferner sicher, dass das bildungspolitische Gesamtkonzept nicht als singuläres Element im kommunalen Raum steht, sondern mit weiteren in der Kommune existenten Gesamtkonzepten bzw. Leitlinien verzahnt wird.

Auf der Grundlage und Etablierung eines Systems einer kommunalen Bildungsberichterstattung sind auf kleinräumiger Ebene die Daten und Planungsbefunde insbesondere aus den Bereichen der Kinder- und Jugendhilfe, der Schule und Bildung und der Sozialstruktur auszuwerten. Danach können unter kommunaler Verantwortung die gemeinsamen bildungs- und jugendhilfepolitischen Ziele mit allen an dem Prozess der Bildung, Erziehung und Betreuung Beteiligten diskutiert und die strategischen Konzepte zu ihrer Erreichung vereinbart werden.

Die mit Kommunalen Bildungslandschaften verbundene verantwortliche Gestaltung aller Angebote der Bildung, Erziehung und Betreuung und die Einbeziehung der Familie als primärer Bildungsort eines jeden jungen Menschen beinhalten neue fachliche Anforderungen an die Kooperationspraxis die Akteure und Institutionen.

Hilfreich bei der Gestaltung der Kooperationsbezüge und des Kooperationsprozesses ist das Wissen um die zentralen Ziele der Bemühungen: das Schaffen von gleichen Bildungsbedingungen und die Herstellung von Chancengerechtigkeit für alle jungen Menschen. Das Handeln der Beteiligten muss sich daher nicht nur durch eine generelle Kooperationsbereitschaft, sondern auch durch eine Bereitschaft zur Verantwortungsübernahme für die Verbesserung von Lebens- und Lernwelten auszeichnen.

Der Deutsche Verein für öffentliche und private Fürsorge wird diesen Prozess der Neujustierung des Bildungssystems weiterhin begleiten und Empfehlungen erarbeiten, die den Aufbau Kommunaler Bildungslandschaften und die Vernetzung der beteiligten Akteure befördern sollen. Ferner ist für das Jahr 2009 eine Fachtagung geplant, die den Beitrag Kommunaler Bildungslandschaften zur Integration junger Menschen mit Migrationshintergrund in das Bildungssystem fokussiert.

\section{Anmerkungen}

(1) Vgl. insbesondere der 8. und der 12. Kinder- und Jugendbericht.

(2) Zur Vertiefung vgl.: Faltermeier, Josef/Mund, Petra: Kommunale Bildungslandschaften - gestalten, in: Archiv für Wissenschaft und Praxis sozialer Arbeit, 39. Jahrgang 3/2008 S. 36-45.

\section{Literatur}

Bundesministerium für Familie, Senioren, Frauen und Jugend (Hg.): Der 12. Kinder- und Jugendbericht der Bundesregierung, Berlin 2005.

Autorengruppe Bildungsberichterstattung (Hg.): Bildung in Deutschland, Bielefeld 2008.

Deutscher Verein für öffentliche und private Fürsorge: Diskussionspapier zum Aufbau Kommunaler Bildungslandschaften, Berlin 2007 (abrufbar im Internet: http://www.deutscher-verein.de).

EU-Kommission: Arbeitsdokument Lebenslanges Lernen: Praxis und Indikatoren, Brüssel 2002.

Faltermeier, Josef/Mund, Petra: Kommunale Bildungslandschaften - gestalten, in: Archiv für Wissenschaft und Praxis sozialer Arbeit, 39. Jahrgang 3/2008 S. 36-45.

Hartnus, Birger; Maykus, Stefan: Schulbezogene Angebote der Jugendhilfe im KJHG, in den Ausführungsgesetzen sowie den Schulgesetzen der Länder. Begründungen und Vorschlag einer bundesrechtlichen Neuverortung im KJHG, in: Hartnus, Birger; Maykus, Stefan (Hg.): Handbuch Kooperation von Jugendhilfe und Schule, Ein Leitfaden für Praxisreflexion, theoretische Verortungen und Forschungsfragen, Berlin 2004, S. 570-592.

Zusammenarbeit von Schule und Jugendhilfe zur »Stärkung und Weiterentwicklung des Gesamtzusammenhangs von Bildung, Erziehung und Betreuung «. Beschluss der Jugendministerkonferenz vom 13. und 14. Mai 2004, Beschluss der Kultusministerkonferenz vom 3. und 4. Juni 2004. 